\title{
Tongue Bracing Under Bite Block Perturbation
}

\author{
Sophia Luo $^{\star 1}$, Yadong Liu ${ }^{\dagger 2}$, Arian Shamei ${ }^{\ddagger 2}$, Murray Schellenberg ${ }^{\star 2}$, Monika Luszczuk ${ }^{\sharp 3}$, and Bryan Gick ${ }^{\nabla 2,4}$ \\ ${ }^{1}$ University of California, Los Angeles, California, USA \\ ${ }^{2}$ University of British Columbia, Vancouver,British Columbia, Canada \\ ${ }^{3}$ Maria Curie-Skłodowska University, Lublin, Poland \\ ${ }^{4}$ Haskins Laboratories, New Haven, Connecticut USA
}

\section{Introduction}

Tongue bracing against the teeth or palate is a pervasive posture maintained continually during normal speech [1]. However, it is unknown whether this bracing is truly necessary for speech, or it is simply a convenient resting place for the sides of the tongue while the jaw is in a relatively high position during speech. The present study aims to test whether the tongue adapts and maintains its bracing position to provide a single, postural parametric mechanism even when the jaw is held open using a bite block. Speech sounds have been shown to adapt quickly but imperfectly under bite block perturbation [2] [3]. When bite blocks translate the jaw (and thereby the tongue) downward, substantial distance is created between the tongue and the hard palate/upper molars, such that bracing would require active upward movement of the sides of the tongue.

Results of an experiment are presented in which native English-speaking participants read aloud a passage under two bite block conditions. Intraoral video results are reported, measuring positions of the lateral tongue for indications of stable bracing postures. In addition, a biomechanical simulation was conducted to help clarify the role of active muscle engagement under bite block conditions. Implications of these findings will be discussed for models of speech production.

\section{Bite block experiment}

\subsection{Methods}

In the present study, video footage was recorded while six native English-speaking participants read aloud an approximately one-minute-long passage under two bite block conditions. The passage was designed with no labial sounds in order to avoid the lips obstructing the camera view. $5 \mathrm{~mm}$ and $10 \mathrm{~mm}$ bite blocks were constructed using wooden tongue depressors, and a small LED light was attached to each bite block to illuminate the oral cavity.

Each participant was seated with the head stabilized. Two bite blocks were placed in the mouth, with one on each side held by the top and bottom back molars. A camera (Sony Cyber-shot DSC-RX100) was positioned directly in front of the mouth, and was manually focused on the upper back molars. For each trial, the participant was first asked to

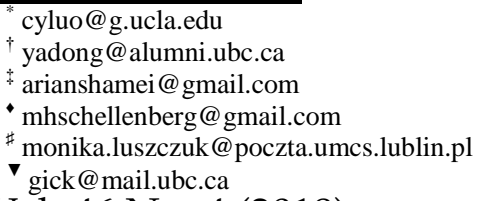

54 - Vol. 46 No. 4 (2018) read the passage twice with $5 \mathrm{~mm}$ bite blocks, then twice again with $10 \mathrm{~mm}$ bite blocks. For each reading, the video recording was started a few seconds before the participant began in order to get footage of the rest position.

For each participant, the token with better imaging quality was selected for each condition, and the 30fps video was converted into an image sequence. We then used the software ImageJ [4] to produce kymographs for the left, right, and center of the tongue (see Figure 1). Each kymograph represents the activity over time through one slice of the video. For the middle of the tongue, the slice was taken between the central incisors; for each side of the tongue, the slice was taken at the back molar.

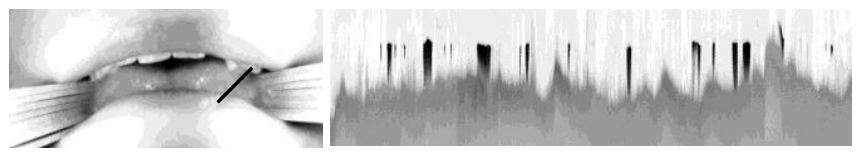

Figure 1: A frame of the video (left), with the black line indicating the location and angle of the slice used to produce the kymograph (right). Each column of pixels corresponds to one frame of the video.

Each kymograph was then turned into non-grayscale black-and-white: light areas correspond to the tongue and teeth, and dark areas correspond to the back of the oral cavity, which is visible when there is no contact between the tongue and palate/teeth. Then, the beginning and end of the kymograph was cropped so that the remaining portion corresponds to active speech. Lastly, a Python script was used to count the number of black pixels in each column of the kymograph, which corresponds to the amount of release from bracing position in one frame of the video. The number of columns that do not contain black pixels corresponds to the duration of contact.

\subsection{Results}

The amount of time during which the tongue was in contact with the upper teeth/palate was converted into percentages (see Figure 2). The results show that the sides of the tongue are almost always in contact with the upper teeth/palate across all speakers regardless of the size of the bite block. However, the center of the tongue does not adopt any such pervasive and parametric posture.

Further, it can be observed that the lateral bracing posture can be interrupted for the production of lateral liquids and some low vowels. Figure 3 shows the sides of the tongue moving away from the braced posture when producing some instances of the lateral liquid [1] and the low vowels [a] and [a].

Canadian Acoustics / Acoustique canadienne 


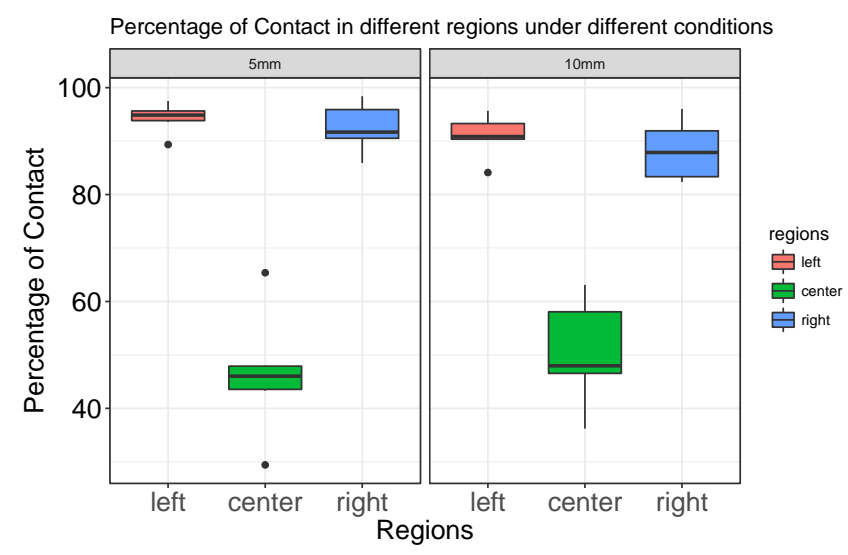

Figure 2: Duration of contact with the upper teeth/palate for different regions of the tongue.

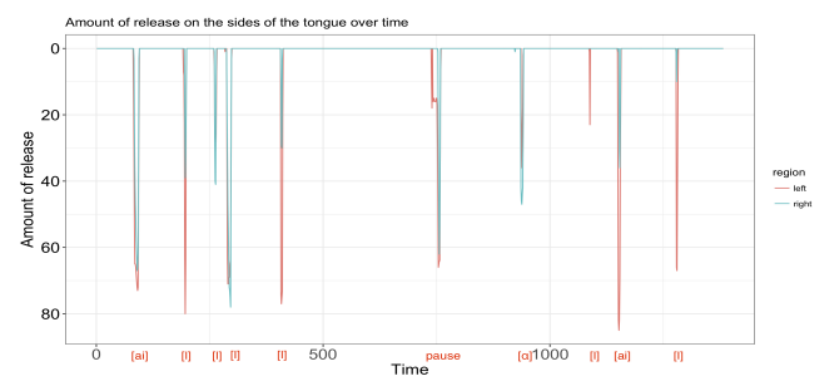

Figure 3: Tracking of the vertical movement of one side of the tongue for one participant. The dips indicate movement away from the bracing posture.

\subsection{Experiment discussion}

The finding that lateral bracing is maintained during speech production under perturbation from bite blocks of different sizes indicates that speakers use bracing as a parametric, intentional, and pervasive mechanism in speech production, in line with Gick et al. (2017). We conducted a biomechanical simulation to further support this point.

\section{Biomechanical simulation}

Muscle-driven forward-dynamic simulations were employed to assess the minimal degree of muscle activation necessary to achieve bracing during natural speech (control condition) and during speech with downward jaw and tongue translation (bite-block conditions). The present analysis uses a 3D finite-element model of the tongue developed by Buchaillard, Perrier, and Payan (2009) [5], with physical parameters mirroring those of Gick et al., (2017). We observed that the minimally-sufficient muscle activation to achieve bracing in natural speech required substantial increases in order to obtain bilateral contact under bite block conditions. Table 1 provides the degree of muscle activation for bilateral openers of the jaw and the minimally-sufficient muscle activation to achieve contact in both conditions. Figure 4 provides illustrations of the simulation using coronal sections from a posterior perspective. Visible artifacts in the tongue illustrations result from sectioning.
Table 1: Minimum activation for contact in each condition.

\begin{tabular}{cccccc}
\hline & JAWO & GGP & MH & VERT & SL \\
\hline Control & $4 \%$ & $20 \%$ & $20 \%$ & $30 \%$ & $30 \%$ \\
Bite Block & $6 \%$ & $30 \%$ & $40 \%$ & $30 \%$ & $60 \%$ \\
\hline & & & & & \\
\hline
\end{tabular}

Figure 4: Bilateral contact in control (left), no contact without increased muscle activation in BB condition (center), and contact following minimally-increased muscle activation (right).

\section{Discussion}

Although lateral bracing under bite block conditions requires significant effort to raise the tongue, speakers still consistently maintain a braced posture with both $5 \mathrm{~mm}$ and $10 \mathrm{~mm}$ bite blocks. Furthermore, although lateral bracing is not required for the production of lateral consonants and low vowels, bracing is often maintained through these speech sounds, which means that bracing is likely the default parameter, while release occurs only when necessary.

\section{Conclusion}

The present study shows that lateral bracing is actively maintained under different degrees of jaw perturbation, suggesting that bracing is a crucial component of speech production. In view of this, it is worth reconsidering current conventions of representing the tongue, which are heavily biased towards the midsagittal plane, and therefore overlook phenomena occurring off-midline. Considering that bracing is indeed a parametric speech posture that continually underlies speech production, future work should consider its implications more broadly for areas such as phonetics, phonology, sound change, clinical practice, and so on.

\section{Acknowledgments}

This project was made possible by funding from NSERC Discovery grant RGPIN-2015-05099 to B. Gick.

\section{References}

[1] B. Gick, B. Allen, F. Roewer-Després, and I. Stavness. Speaking tongues are actively braced. JSLHR, 60:494, 2017.

[2] T. Gay, B. Lindblom, and J. Lubker. Production of bite-block vowels: acoustic equivalence by selective compensation. JASA, 69:802, 1981.

[3] J. E. Flege, S. G. Fletcher, and A. Homiedan. Compensating for a bite block in /s/ and /t/ production: palatographic, acoustic, and perceptual data. JASA, 83:212, 1988.

[4] C. A. Schneider, W. S. Rasband, and K. W. Eliceiri. NIH Image to ImageJ: 25 years of image analysis, Nature methods, 9(7):671-5, 2012.

[5] S. Buchaillard, P. Perrier, and Y. Payan. A biomechanical model of cardinal vowel production: muscle activations and the impact of gravity on tongue positioning. JASA, 126(4):2033-51, 2009. 